\title{
Oral beta-lactam step down in bacteremic E. coli urinary tract infections
}

Stephan Saad ${ }^{1 *}$ (D), Neil Mina ${ }^{2}$, Colin Lee ${ }^{3}$ and Kevin Afra ${ }^{4^{*}}$

\begin{abstract}
Background: Literature is scarce regarding oral step down to beta-lactams in bacteremic urinary tract infections. Oral fluoroquinolones are an accepted and common step down for bacteremic urinary tract infections; however, their use is associated with mounting safety concerns. We compared clinical cure in patients with E. coli bacteremic urinary tract infections who were stepped down to oral beta-lactams compared to oral fluoroquinolones.

Methods: This multicentre retrospective cohort study included patients with first positive concurrent urine and blood cultures from January 2016 to December 2016. Patients were included if they received empiric intravenous beta-lactam therapy with step down to either oral beta-lactam or fluoroquinolone for treatment completion. The primary outcome was clinical cure. Secondary outcomes were length of hospitalization, all-cause mortality and $C$. difficile infection. Multivariate analysis and propensity score were used to control for confounding.

Results: A total of 207 patients were identified with bacteremic E.coli urinary tract infections. Clinical cure was achieved in 72/77 (94\%) in the oral beta-lactam group versus 127/130 (98\%) in the oral fluoroquinolone group (absolute difference $-4.2,95 \%$ confidence interval $[\mathrm{Cl}]-10.3$ to $1.9 \%, p=0.13$ ). The adjusted odds ratio (OR) for clinical cure with oral beta-lactams was 0.31 (95\% Cl 0.05-1.90, $p=0.21$ ); propensity score adjusted analysis showed a similar result. There was no statistically significant difference in secondary outcomes.
\end{abstract}

Conclusions: Oral beta-lactams appear to be a safe and effective step down option in bacteremic $E$. coli urinary tract infections compared to oral fluoroquinolones.

Keywords: Oral step down, Antibiotics, E. coli, Gram negative bacteremia, Urinary tract infection

\section{Summary}

Identifying effective alternatives to fluoroquinolones is important given mounting recent safety concerns. This retrospective study finds no difference in clinical outcomes for oral beta-lactam step down in bacteremic E. coli urinary tract infections compared to oral fluoroquinolones.

\footnotetext{
* Correspondence: stephan.saad@alumni.ubc.ca; kevin.afra@fraserhealth.ca 'Department of Medicine, University of British Columbia, 2733 Heather Street, Room C328, Vancouver, British Columbia V5Z 3J5, Canada

${ }^{4}$ Department of Medicine, University of British Columbia, Suite 400-13450 102 Ave, Surrey, British Columbia V3T 5X4, Canada

Full list of author information is available at the end of the article
}

\section{Background}

Pyelonephritis is a common indication for antibiotic use with an incidence of $5-15$ cases per 10,000 population [1]. E. coli is the most common causative organism, responsible for up to $80 \%$ of cases $[1,2]$. Treatment guidelines for pyelonephritis have historically favoured use of fluoroquinolones for oral therapy [3]. Oral beta-lactams are thought to be less effective. However, increasing resistance among uropathogens like E. coli to fluoroquinolones necessitate a reassessment of the role of oral beta-lactams in management of pyelonephritis. Moreover, regulatory agencies have released multiple recent warnings about the underappreciated harms of fluoroquinolones [4-10].

(c) The Author(s). 2020 Open Access This article is licensed under a Creative Commons Attribution 4.0 International License, which permits use, sharing, adaptation, distribution and reproduction in any medium or format, as long as you give appropriate credit to the original author(s) and the source, provide a link to the Creative Commons licence, and indicate if changes were made. The images or other third party material in this article are included in the article's Creative Commons licence, unless indicated otherwise in a credit line to the material. If material is not included in the article's Creative Commons licence and your intended use is not permitted by statutory regulation or exceeds the permitted use, you will need to obtain permission directly from the copyright holder. To view a copy of this licence, visit http://creativecommons.org/licenses/by/4.0/ The Creative Commons Public Domain Dedication waiver (http://creativecommons.org/publicdomain/zero/1.0/) applies to the data made available in this article, unless otherwise stated in a credit line to the data. 
In pediatric patients with pyelonephritis, oral betalactams have already been established as a safe and effective treatment option. Canadian guidelines recommend that oral antibiotics be used as initial management of pyelonephritis, unless the child is toxic or has known structural urologic abnormalities [11].

Oral beta-lactam step down in pyelonephritis was reported routinely during randomized trials in the 1990's evaluating pyelonephritis management in pregnancy [12-15]. Only one of these trials directly compared intravenous to oral beta-lactam therapy, finding no difference in clinical success; however, bacteremia was excluded [12]. Several further trials in non-pregnant women with pyelonephritis have shown equivalent efficacy of oral beta-lactams in step down compared with ongoing intravenous therapy $[16,17]$. Bacteremia was uncommon in these trials (20-25\%), raising doubts about whether results could be extrapolated to patients with bacteremic urinary tract infections. Moreover, very little has been published in treatment of adult males with bacteremic pyelonephritis.

We sought to compare the clinical outcomes of adult patients with urinary tract infection and $E$. coli bacteremia who received oral beta-lactam step down compared to oral fluoroquinolone following initial empiric intravenous beta-lactam therapy.

\section{Methods}

\section{Study design}

We conducted a multicentre retrospective cohort study including patients with first positive concurrent blood and urine cultures for E. coli from January 1, 2016 to December 31, 2016 within Fraser Health, which serves 1.8 million people in British Columbia, Canada. The study was approved by Fraser Health Research Ethics Board (FHREB File \#: 2017-015).

Patients with $E$. coli positive blood and urine cultures were identified from an electronic microbiology database. Patients were included in this study if they were 18 years or older and had both blood and urine cultures positive for $E$. coli within $72 \mathrm{~h}$ of each other. Patients were only included if they received empiric intravenous beta-lactam therapy and followed by step down to oral beta-lactam or oral fluoroquinolone for treatment completion. Timing and agent of step down therapy was at the discretion of the treating physician. Patients who did not survive for at least $72 \mathrm{~h}$ after first positive blood culture were excluded.

Patients were also excluded for the following reasons: non-urinary source of $E$. coli bacteremia as documented by the treating physician; treatment for a concomitant infection; presence of complicated pyelonephritis identified within first 7 days of therapy (emphysematous pyelonephritis, renal parenchymal abscess, perinephric abscess, renal papillary necrosis); prostatitis; extended spectrum beta-lactamase producing, AmpC, or carbapenem-resistant E. coli; HIV with CD4<200; neutropenia with $\mathrm{ANC}<0.5$; or renal transplant.

Demographic, clinical and outcome data were acquired from electronic medical records. Charlson co-morbidity index and Pitt bacteremia score data were collected at baseline. Sepsis and septic shock was defined using the Sepsis-3 international consensus definitions [18]. Infections were classified as community-acquired healthcareassociated infections if any of the following criteria were met: Living in a long-term care facility or nursing home, receiving outpatient intravenous therapy within the past 30 days, attending a hospital or hemodialysis clinic within the past 30 days or being admitted for more than 2 days within the past 90 days. A full list of definitions can be found in Additional file 1.

\section{Outcome measures}

The primary outcome was clinical cure defined by meeting all of the following criteria: resolution or improvement of symptoms during treatment, no recurrence of symptoms or signs of urinary tract infection within 30 days, and no discontinuation or change of treatment because of worsened or persistent symptoms or occurrence of adverse events.

Secondary outcomes included length of hospitalization following first positive blood culture, all-cause mortality within 30 days of first positive blood culture, and C. difficile positive stool sample within 30 days of first positive blood culture.

\section{Microbiology methods}

Blood and urine cultures were processed by a centralized clinical microbiology laboratory located within Fraser Health. Antibiotic susceptibility was determined by the Vitek 2 System (bioMérieux, Laval, Quebec). Clinical and Laboratory Standards Institute breakpoints were used to determine antimicrobial susceptibility breakpoints (2016 M100 26th edition).

\section{Statistical analysis}

Categorical variables were analyzed using Pearson chisquared or Fisher's exact test. Continuous variables were analyzed using Mann Whitney U test. Statistical significance for all tests was set at a p-value of less than 0.05 .

Association between antibiotic therapy (oral betalactam or oral fluoroquinolone) and clinical cure was modelled using logistic regression and expressed as odds ratios (ORs) and 95\% confidence intervals (CI). Additional adjustment for covariates with a $P$ value of $<0.20$ on univariate analysis were included in the adjusted logistic regression model. 
Further analysis using propensity score was performed to account for potential residual confounding. We constructed a propensity score for receiving antibiotic therapy (oral beta-lactam or oral fluoroquinolone) as the dependent variable using logistic regression modelling. Covariates included in generating the propensity score included age, sex, diabetes mellitus, chronic kidney disease, heart conditions, immune compromise, recurrent UTI risk factors (history of recurrent UTI, anatomical or functional urinary tract abnormalities, or nephrolithiasis), Pitt bacteremia score, sepsis, beta-lactam allergy, and duration of intravenous therapy. A final analysis using logistic regression to assess for association between antibiotic therapy and clinical cure was performed using propensity score as a single covariate. Statistical analyses were performed using STATA IC version 13.1 and RStudio version 1.2.503.

\section{Results}

There were 207 patients who met eligibility criteria during the study period. One hundred and thirty patients $(62.8 \%)$ were stepped down to an oral fluoroquinolone, while 77 (37.2\%) were stepped down to an oral betalactam. Baseline clinical and demographic data are presented in Table 1. Patients stepped down to an oral beta-lactam were more likely to have acquired the UTI in a community acquired health-care associated setting (26\% vs $11.5 \%$ ) and less likely in a community acquired setting (68.8\% vs $84.6 \%)$. All other baseline factors were similar between groups, including indicators for severity of infection such as sepsis or septic shock, ICU admission, and Pitt bacteremia score. The median number of days of intravenous treatment was similar between patients stepped down to oral beta-lactam compared to oral fluoroquinolone ( 5 vs 5 days, $p=0.20$ ), as was oral treatment duration ( 7 vs 7 days, $p=0.38$ ), and total treatment duration (14 vs 14 days, $p=0.91$ ).

The primary outcome of clinical cure was achieved in 127 patients (98\%) in the oral fluoroquinolone group and in 72 patients $(94 \%)$ in the oral beta-lactam group (absolute difference $-4.2,95 \% \mathrm{CI},-10.3$ to $1.9 \%, p=$ 0.13) (Table 2).

Unadjusted analysis demonstrated no significant difference between oral beta-lactam and oral fluoroquinolone groups for clinical cure (OR 0.34; 95\% CI, 0.08-1.47, $p=$ 0.15) (Table 3). Results were similar using multivariate adjustment (adjusted OR, 0.31; 95\% CI, 0.05-1.90, $p=$ 0.2 ) and propensity scoring adjustment (OR, 0.31; $95 \%$ CI, 0.07-1.38, $p=0.12$ ). Female sex was not associated with higher clinical cure (OR 2.49; 95\% CI, 0.60-10.30, $p=0.21$ ).

The median length of hospitalization was 6 days in both groups $(p=0.43)$. There was one mortality in the oral fluoroquinolone group, and one case of $C$. difficile in each group; neither of these secondary outcomes were statistically significant.

Patients stepped down to an oral fluoroquinolone were all prescribed ciprofloxacin (Table 4). Conversely, a variety of antibiotics were used as step down for those given an oral beta-lactam, including amoxicillin, amoxicillinclavulanate, cephalexin, cefuroxime, and cefixime.

\section{Discussion}

Our results suggest that oral beta-lactams are a safe and effective step down for patients with $E$. coli bacteremic urinary tract infections. At baseline, the two treatment groups had similar co-morbidities, severity of infection and duration of intravenous treatment. In this population of bacteremic patients, there was no difference in rates of clinical cure or length of hospitalization. Approximately $30 \%$ of patients in our study were male with no significant difference in clinical cure rate compared to females. Mortality and $C$. difficile infection were infrequent occurrences, with no difference between the groups.

This study isolated a group of bacteremic patients with pyelonephritis to demonstrate the suitability of oral beta-lactam step down and adds to a growing body of literature with similar conclusions. A randomized controlled trial by Sanchez et al. compared daily intravenous ceftriaxone to single dose intravenous ceftriaxone followed by oral cefixime, both to total 10 days of treatment [16]. Their study included 144 women with uncomplicated pyelonephritis. E. coli was the responsible organism in nearly all cases and $25 \%$ of patients had bacteremia. There was no difference in clinical cure between groups (91\% vs 92\% respectively). Another randomized controlled trial by Monmaturapoj et al. evaluated daily intravenous ceftriaxone to 3 days of intravenous ceftriaxone followed by oral cefditoren, both to total 10 days of treatment [17]. Their study included 82 patients with pyelonephritis, only three of whom were male. E. coli was identified in $84 \%$ of cases and $21 \%$ of patients had bacteremia. Again, no difference in clinical cure rate was found (95\% vs 100\% respectively). Both of these randomized controlled trials were limited by the small number of male patients and minority of cases with bacteremia.

A recent observational study by Tamma et al. showed similar 30-day mortality in a cohort of hospitalized patients with Enterobacteriaceae bacteremia who received oral step down therapy compared to ongoing intravenous therapy [19]. Nearly half the patients in their study were male. A wide range of infections were included, with urinary tract infection comprising 36.5\%. However, fluoroquinolones were the most commonly used oral step down therapy $(70 \%)$, while oral beta-lactams were only used in a minority (16.5\%). This reflects the 
Table 1 Baseline characteristics of patients

\begin{tabular}{|c|c|c|c|}
\hline & $\begin{array}{l}\text { Oral fluoroquinolone step } \\
\text { down }(n=130)\end{array}$ & $\begin{array}{l}\text { Oral beta-lactam step } \\
\text { down }(n=77)\end{array}$ & $p$-value \\
\hline Age, median, years (IQR) & $70.5(56.25-80.75)$ & $71(54-80)$ & 0.72 \\
\hline Sex & & & 0.92 \\
\hline Female & $92(71)$ & $54(70)$ & \\
\hline Male & $38(29)$ & $23(30)$ & \\
\hline \multicolumn{4}{|l|}{ Comorbidities } \\
\hline Diabetes Mellitus & $29(22)$ & $20(26)$ & 0.55 \\
\hline Heart Conditions & $75(58)$ & $43(56)$ & 0.8 \\
\hline Chronic Kidney Disease & $10(8)$ & $3(4)$ & 0.28 \\
\hline Peripheral Vascular Disease & $6(5)$ & $4(5)$ & 0.85 \\
\hline Asthma or COPD & $14(11)$ & $7(9)$ & 0.7 \\
\hline Liver Cirrhosis & $1(1)$ & $2(3)$ & 0.56 \\
\hline Recurrent UTI Risk factors & $38(29)$ & $22(29)$ & 0.92 \\
\hline IVDU & $5(4)$ & $0(0)$ & 0.16 \\
\hline Hepatitis C & $1(1)$ & $1(1)$ & 1.0 \\
\hline HIV & $0(0)$ & $0(0)$ & 1.0 \\
\hline Immunocompromised & $5(4)$ & $4(5)$ & 0.65 \\
\hline Charlson comorbidity index, median (IQR) & $1(0-2)$ & $1(0-3)$ & 0.96 \\
\hline Catheter associated UTI & $6(5)$ & $4(5)$ & 0.85 \\
\hline Hospital admission & $101(78)$ & $62(81)$ & 0.63 \\
\hline ICU admission & $9(7)$ & $5(6)$ & 0.91 \\
\hline Sepsis & $31(25)$ & $25(33)$ & 0.2 \\
\hline Septic shock & $8(6)$ & $5(6)$ & 0.92 \\
\hline Pitt bacteremia score, median (IQR) & $0.5(0-1)$ & $1(0-1)$ & 0.53 \\
\hline Site of acquisition & & & 0.02 \\
\hline Community-acquired & $110(85)$ & $53(69)$ & \\
\hline Community-acquired health-care associated & $15(12)$ & $20(26)$ & \\
\hline Hospital-acquired & $5(4)$ & $4(5)$ & \\
\hline Beta-lactam allergy & $15(12)$ & $7(9)$ & 0.58 \\
\hline Inadequate empiric treatment & $1(1)$ & $1(1)$ & 1.0 \\
\hline Stable at oral switch & $125(96)$ & $74(96)$ & 0.99 \\
\hline Duration of intravenous treatment, median, days (IQR) & $5(3-7)$ & $5(3-7)$ & 0.20 \\
\hline Duration of oral treatment, median, days (IQR) & $7(7-10)$ & $7(5.75-10)$ & 0.38 \\
\hline Total treatment duration, median, days (IQR) & $14(12-14)$ & $14(11-14)$ & 0.91 \\
\hline
\end{tabular}

Data are number (percentage) of patients unless otherwise indicated

Abbreviations: COPD chronic obstructive pulmonary disease, ICU intensive care unit, IVDU intravenous drug use, UTI urinary tract infection;

widespread practice of avoiding oral beta-lactams in step down therapy for gram negative bacteremia.

Mercuro et al. compared fluoroquinolones to betalactams in oral step down for Enterobacteriaceae bacteremia [20]. Over $50 \%$ of patients were male. E. coli was the dominant organism (71\%), with approximately $70 \%$ having a urinary tract infection source. No difference in clinical success was noted between the two groups. However, their study included only admitted patients. Over $20 \%$ of patients in our study were managed as outpatients, without hospital admission. Furthermore, including a broad range of infections may mask differences between individual subgroups (e.g., urinary tract infections). As a homogenous patient population of bacteremic urinary tract infections our study is not affected by this limitation.

A recent meta-analysis by Punjabi et al. found no difference in all-cause mortality between oral beta-lactam and oral fluoroquinolones in step down of Enterobacteriaceae bacteremia [21]. Oral beta-lactams appeared to 
Table 2 Thirty-day outcomes for patients

\begin{tabular}{|c|c|c|c|}
\hline & $\begin{array}{l}\text { Oral fluoroquinolone step } \\
\text { down }(n=130)\end{array}$ & $\begin{array}{l}\text { Oral beta-lactam step } \\
\text { down }(n=77)\end{array}$ & $p$-value \\
\hline \multicolumn{4}{|l|}{ Primary Outcome } \\
\hline Clinical cure & $127(98)$ & $72(94)$ & 0.13 \\
\hline \multicolumn{4}{|l|}{ Secondary Outcomes } \\
\hline 30-day mortality & $1(1)$ & $0(0)$ & 0.43 \\
\hline CDI & $1(1)$ & $1(1)$ & 1 \\
\hline Length of hospitalization, median, days (IQR) & $6(3.25-9)$ & $6(4-10)$ & 0.43 \\
\hline
\end{tabular}

Data are number (percentage) of patients unless otherwise indicated

Abbreviation: CDI Clostridioides difficile infection

have higher rates of infection recurrence compared to fluoroquinolones. The authors hypothesize that underdosing of beta-lactams may be contributing to this finding. Including a wide range of source infections confounds this interpretation.

The impetus to limit fluoroquinolone prescribing is growing. Fluoroquinolone prescribing is an established risk for $C$. difficile infection, both at the individual and community level $[22,23]$. Multiple statements have been issued by Health Canada, the United States Food and Drug Administration, and the European Medicines Association warning about serious and underappreciated side effects [4-10]. For fluoroquinolone prescribing to be curbed, other therapeutic options with similar efficacy and improved safety profile need to be identified. Furthermore, despite the side effects of fluoroquinolones, they fulfil a critical role as oral therapy for difficult to treat gram-negative infections due to Pseudomonas or Acinetobacter. Limiting fluoroquinolone use to these infections where other suitable oral alternatives do not exist is prudent antimicrobial stewardship.

Fluoroquinolones have long been regarded as an effective agent for bacteremic infections. Pharmacologic factors including high oral bioavailability, convenient dosing, and ability to attain dosing targets have supported this practice. This is supported by a large body of controlled literature and extensive real life clinical experience. Oral beta-lactams do not share the same

Table 3 Univariate and multivariate analysis for thirty-day clinical cure in E. coli bacteremic urinary tract infections

\begin{tabular}{|c|c|c|c|c|}
\hline \multirow[t]{2}{*}{ Variable } & \multicolumn{2}{|c|}{ Unadjusted Analysis } & \multicolumn{2}{|c|}{ Multivariate Analysis } \\
\hline & OR $(95 \% \mathrm{Cl})$ & $p$-value & OR $(95 \% \mathrm{Cl})$ & $p$-value \\
\hline Age (per year) & $0.98(0.93-1.02)$ & 0.30 & $\ldots$ & $\ldots$ \\
\hline Sex (female) & $2.49(0.60-10.30)$ & 0.21 & $\ldots$ & $\ldots$ \\
\hline DM & $0.29(0.07-1.22)$ & 0.09 & $0.50(0.08-3.04)$ & 0.45 \\
\hline CKD & $0.18(0.03-0.97)$ & 0.046 & $0.28(0.03-3.06)$ & 0.30 \\
\hline Heart Conditions & $0.18(0.02-1.49)$ & 0.11 & $0.38(0.03-4.43)$ & 0.44 \\
\hline Asthma/COPD & $0.78(0.09-6.69)$ & 0.82 & $\ldots$ & $\ldots$ \\
\hline Immunocompromised & $0.29(0.03-2.68)$ & 0.28 & $\ldots$ & $\ldots$ \\
\hline Recurrent UTI risk factors & $0.12(0.02-0.63)$ & 0.01 & $0.15(0.02-0.96)$ & 0.045 \\
\hline CA-UTI & $0.33(0.04-2.99)$ & 0.33 & $\ldots$ & $\ldots$ \\
\hline Charlson (per unit) & $0.69(0.52-0.92)$ & 0.01 & $0.71(0.41-1.21)$ & 0.20 \\
\hline Pitt (per unit) & $0.72(0.49-1.04)$ & 0.08 & $0.66(0.39-1.10)$ & 0.11 \\
\hline Sepsis & $0.61(0.14-2.62)$ & 0.50 & $\ldots$ & $\ldots$ \\
\hline Septic Shock & $0.45(0.05-3.95)$ & 0.47 & $\ldots$ & $\ldots$ \\
\hline ICU admission & $0.49(0.05-4.28)$ & 0.52 & $\ldots$ & $\ldots$ \\
\hline Beta-lactam allergy & $0.34(0.06-1.77)$ & 0.20 & $0.14(0.02-1.14)$ & 0.07 \\
\hline IV therapy duration & $0.83(0.67-1.03)$ & 0.09 & $0.88(0.65-1.18)$ & 0.40 \\
\hline Total duration & $1.01(0.80-1.27)$ & 0.96 & $\ldots$ & $\ldots$ \\
\hline Oral beta-lactam step down & $0.34(0.08-1.47)$ & 0.15 & $0.31(0.05-1.90)$ & 0.21 \\
\hline
\end{tabular}

Abbreviations: CA-UTI catheter-associated urinary tract infection, CKD chronic kidney disease, Cl confidence interval, COPD chronic obstructive pulmonary disease, $D M$ diabetes mellitus, ICU intensive care unit, IV intravenous, OR odds ratio, UTI urinary tract infection 
Table 4 Oral antibiotic step down regimens

\begin{tabular}{|c|c|c|c|}
\hline & Antibiotic & Regimen & Frequency (\%) \\
\hline \multirow[t]{6}{*}{ Oral fluoroquinolone step down $(n=130)$} & Ciprofloxacin & 500 mg po BID & $114(87.7)$ \\
\hline & & 500 mg po daily & $5(3.9)$ \\
\hline & & $250 \mathrm{mg}$ po BID & $5(3.9)$ \\
\hline & & 750 mg po BID & $2(1.5)$ \\
\hline & Ciprofloxacin ER & 1000 mg po daily & $3(2.3)$ \\
\hline & & 500 mg po daily & $1(0.8)$ \\
\hline \multirow[t]{9}{*}{ Oral beta-lactam step down $(n=77)$} & Cefixime & 400 mg po daily & $24(31.2)$ \\
\hline & Cephalexin & $500 \mathrm{mg}$ po QID & $19(24.7)$ \\
\hline & & 1000 mg po QID & $1(1.3)$ \\
\hline & Amoxicillin-clavulanate & 875 mg po BID & $10(13.0)$ \\
\hline & & 500 mg po TID & $7(9.1)$ \\
\hline & & 500 mg po BID & $2(2.6)$ \\
\hline & Amoxicillin & 500 mg po TID & $5(6.5)$ \\
\hline & & 500 mg po BID & $2(2.6)$ \\
\hline & Cefuroxime & 500 mg po BID & $7(9.1)$ \\
\hline
\end{tabular}

Data are number (percentage) of patients unless otherwise indicated

Abbreviation: $E R$ extended release, BID two times a day, TID three times a day, QID four times a day

historical confidence in serious invasive infections including bacteremia. Pharmacokinetic concerns including lower oral bioavailability and more frequent dosing are reason for pause. However, recent randomized controlled trials using oral beta-lactams in treatment of infective endocarditis and complex orthopedic infections challenge these historical dogmas [24, 25].

Our study has several limitations. First, its retrospective design limits our ability to control for confounders to those that were collected. Second, selection bias could have been an issue, as unaccounted patient factors may have led a physician to choose a certain oral step down option, or not to step down to oral therapy at all. The use of propensity score analysis can partly account for this confounding. Third, some relevant outcomes may have been missed. Data were abstracted from the Health Authority electronic medical record, thus potentially relevant outcomes only captured in outpatient records were not accessible. Fourth, the convenience sample of 1 year resulted in a limited sample size. This study may be underpowered to identify a difference between treatment arms where one truly does exist. Fifth, our study excluded complicated cases (e.g., renal abscess) and prostatitis. Our results should not be extrapolated to the whole population of bacteremic urinary tract infections. Lastly, both groups received a median of 5 days of intravenous therapy and 14 days of total treatment. This longer treatment duration may have reduced the chance to find a difference between the groups.

\section{Conclusion}

Future research is needed to further establish the safety, efficacy, and optimal duration of oral step down therapy for bacteremic urinary tract infections, in particular with oral beta-lactams. While observational studies can corroborate our findings, randomized controlled trials would provide clinicians the greatest confidence in using oral beta-lactam therapy as step down for bacteremic urinary tract infections.

In conclusion, oral beta-lactam step down in the setting of urinary tract infections with $E$. coli bacteremia had a similar clinical cure rate to that of oral fluoroquinolone step down. Given the potential adverse effects associated with fluoroquinolones, beta-lactams present a safe and efficacious alternative oral step-down agent.

\section{Supplementary information}

Supplementary information accompanies this paper at https://doi.org/10. 1186/s12879-020-05498-2.

Additional file 1. Definitions of terms used to collect data

\section{Abbreviations}

ORs: Odds ratios; Cl: Confidence intervals; UTI: Urinary tract infection; ICU: Intensive care unit; COPD: Chronic obstructive pulmonary disease; IVDU: Intravenous drug use; CDI: Clostridioides difficile infection; CAUTI: Catheter-associated urinary tract infection; CKD: Chronic kidney disease; DM: Diabetes mellitus; IV: Intravenous; ER: Extended release; BID: Two times a day; TID: Three times a day; QID: Four times a day

Acknowledgements None. 


\section{Authors' contributions}

SS and KA were responsible for formulating the research questions and the methodologic design of the study. SS, NM and KA were responsible for acquisition and interpretation of data. SS and KA performed statistical analyses. SS and KA drafted the manuscript. SS, CL, NM and KA provided critical revision of the manuscript for important intellectual content. KA supervised the project. All authors read and approved the final manuscript.

\section{Funding}

This study was conducted with no external funding.

\section{Availability of data and materials}

The datasets used during the current study are available from the corresponding author on reasonable request.

\section{Ethics approval and consent to participate}

The study was approved by Fraser Health Research Ethics Board. All patient information was anonymized by assigning each subject a unique study code.

\section{Consent for publication}

Not applicable.

\section{Competing interests}

No competing interests to declare.

\section{Author details}

'Department of Medicine, University of British Columbia, 2733 Heather Street, Room C328, Vancouver, British Columbia V5Z 3J5, Canada. ${ }^{2}$ Department of Laboratory Medicine, Fraser Health, 1375096 Ave, Surrey, British Columbia V3V 1Z2, Canada. ${ }^{3}$ Department of Pharmacy, Providence Health, 1081 Burrard St, Vancouver, British Columbia V6Z 1Y6, Canada. ${ }^{4}$ Department of Medicine, University of British Columbia, Suite 400-13450 102 Ave, Surrey, British Columbia V3T 5X4, Canada.

\section{Received: 17 August 2020 Accepted: 12 October 2020}

\section{Published online: 21 October 2020}

\section{References}

1. Czaja CA, Scholes D, Hooton TM, Stamm WE. Population-based epidemiologic analysis of acute pyelonephritis. Clin Infect Dis. 2007;45: 273-80.

2. Zhanel GG, Hisanaga TL, Laing NM, et al. Antibiotic resistance in outpatient urinary isolates: final results from the north American urinary tract infection collaborative Alliance (NAUTICA). Int J Antimicrob Agents. 2005;26:380-8.

3. Gupta K, Hooton TM, Naber KG, et al. International clinical practice guidelines for the treatment of acute uncomplicated cystitis and pyelonephritis in women: a 2010 update by the Infectious Diseases Society of America and the European Society for Microbiology and Infectious Diseases. Clin Infect Dis. 2011;52:e103-20.

4. U.S. Food \& Drug Administration. FDA Drug Safety Communication: FDA advises restricting fluoroquinolone antibiotic use for certain uncomplicated infections; warns about disabling side effects that can occur together. 2016. http://www.fda.gov/drugs/drug-safety-and-availability/fda-drug-safetycommunication-fda-advises-restricting-fluoroquinolone-antibiotic-usecertain. Accessed 27 May 2019

5. U.S. Food \& Drug Administration. FDA Drug Safety Communication: FDA updates warnings for oral and injectable fluoroquinolone antibiotics due to disabling side effects. 2016. http://www.fda.gov/drugs/drug-safety-andavailability/fda-drug-safety-communication-fda-updates-warnings-oral-andinjectable-fluoroquinolone-antibiotics. Accessed 27 May 2019.

6. U.S. Food \& Drug Administration. FDA reinforces safety information about serious low blood sugar levels and mental health side effects with fluoroquinolone antibiotics; requires label changes. 2018. http:/www.fda. gov/drugs/drug-safety-and-availability/fda-reinforces-safety-informationabout-serious-low-blood-sugar-levels-and-mental-health-side. Accessed 27 May 2019.

7. U.S. Food \& Drug Administration. FDA warns about increased risk of ruptures or tears in the aorta blood vessel with fluoroquinolone antibiotics in certain patients. 2018. http://www.fda.gov/drugs/drug-safety-andavailability/fda-warns-about-increased-risk-ruptures-or-tears-aorta-bloodvessel-fluoroquinolone-antibiotics. Accessed 27 May 2019.
8. Health Canada. Summary safety review - Oral FLUOROQUINOLONES assessing the potential risk of retinal detachment. 2016. https://www. canada.ca/en/health-canada/services/drugs-health-products/medeffectcanada/safety-reviews/summary-safety-review-oral-fluoroquinolonesassessing-potential-risk-retinal.html. Accessed 27 May 2019.

9. Health Canada. Summary safety review - Fluoroquinolones - assessing the potential risk of persistent and disabling side effects. 2017. https://www. canada.ca/en/health-canada/services/drugs-health-products/medeffectcanada/safety-reviews/summary-safety-review-fluoroquinolones-assessingpotential-risk-persistent-disabling-effects.html. Accessed 27 May 2019.

10. European Medicines Agency. Quinolone- and fluoroquinolone-containing medicinal products. 2019. https:/www.ema.europa.eu/en/medicines/ human/referrals/quinolone-fluoroquinolone-containing-medicinal-products. Accessed 27 May 2019

11. Robinson JL, Finlay JC, Lang ME, Bortolussi R. Canadian Paediatric society, infectious diseases and immunization committee, community Paediatrics committee. Urinary tract infections in infants and children: diagnosis and management. Paediatr Child Health. 2014;19:315-25.

12. Angel JL, O'Brien WF, Finan MA, Morales WJ, Lake M, Knuppel RA. Acute pyelonephritis in pregnancy: a prospective study of oral versus intravenous antibiotic therapy. Obstet Gynecol. 1990;76:28-32.

13. Millar LK, Wing DA, Paul RH, Grimes DA. Outpatient treatment of pyelonephritis in pregnancy: a randomized controlled trial. Obstet Gynecol. 1995;86:560-4.

14. Wing DA, Hendershott CM, Debuque L, Millar LK. A randomized trial of three antibiotic regimens for the treatment of pyelonephritis in pregnancy. Obstet Gynecol. 1998;92:249-53.

15. Wing DA, Hendershott CM, Debuque L, Millar LK. Outpatient treatment of acute pyelonephritis in pregnancy after 24 weeks. Obstet Gynecol. 1999;94: 683-8.

16. Sanchez M, Collvinent B, Miró O, et al. Short-term effectiveness of ceftriaxone single dose in the initial treatment of acute uncomplicated pyelonephritis in women. A randomised controlled trial. Emerg Med J. 2002; 19:19-22.

17. Monmaturapoj T, Montakantikul P, Mootsikapun P, Tragulpiankit P. A prospective, randomized, double dummy, placebo-controlled trial of oral cefditoren pivoxil $400 \mathrm{mg}$ once daily as switch therapy after intravenous ceftriaxone in the treatment of acute pyelonephritis. Int J Infect Dis. 2012;16 e843-9.

18. Singer M, Deutschman CS, Seymour CW, et al. The third international consensus definitions for Sepsis and septic shock (Sepsis-3). JAMA. 2016;315: $801-10$.

19. Tamma PD, Conley AT, Cosgrove SE, et al. Association of 30-day mortality with Oral step-down vs continued intravenous therapy in patients hospitalized with Enterobacteriaceae bacteremia. JAMA Intern Med. 2019; 179(3):316-23.

20. Mercuro NJ, Stogsdill P, Wungwattana M. Retrospective analysis comparing oral stepdown therapy for enterobacteriaceae bloodstream infections: fluoroquinolones versus $\beta$-lactams. Int J Antimicrob Agents. 2018;51:687-92.

21. Punjabi C, Tien V, Meng L, Deresinski S, Holubar M. Oral Fluoroquinolone or Trimethoprim-sulfamethoxazole vs. B-lactams as Step-Down Therapy for Enterobacteriaceae Bacteremia: Systematic Review and Meta-analysis. Open Forum Infect Dis. 2019;6:ofz364.

22. Deshpande A, Pasupuleti V, Thota P, et al. Community-associated Clostridium difficile infection and antibiotics: a meta-analysis. J Antimicrob Chemother. 2013;68:1951-61.

23. Dingle KE, Didelot $X$, Quan TP, et al. Effects of control interventions on Clostridium difficile infection in England: an observational study. Lancet Infect Dis. 2017:17:411-21.

24. Iversen K, Ihlemann N, Gill SU, et al. Partial Oral versus intravenous antibiotic treatment of endocarditis. N Engl J Med. 2019:380:415-24.

25. Li H-K, Rombach I, Zambellas R, et al. Oral versus intravenous antibiotics for bone and joint infection. N Engl J Med. 2019;380:425-36.

\section{Publisher's Note}

Springer Nature remains neutral with regard to jurisdictional claims in published maps and institutional affiliations. 